\title{
Control Point
}

National Cancer Institute

\section{Source}

National Cancer Institute. Control Point. NCI Thesaurus. Code C112866.

Any one of a set of linear accelerator parameters that define the segments from which a radiation beam is applied. 